\title{
LA UNIVERSIDAD Y LOS OTROS
}

Patrick Boulet

Docente e Investigador en Instituto de Formación Docente, Argentina.

\section{INTRODUCCIÓN}

La universidad argentina mantiene, al menos en el discurso de mucho de sus actores, una pretensión de universidad popular y abierta a diferentes sectores sociales, postulado que muchas veces no coincide con los datos cuantitativos que desde el gobierno nacional y las mismas casas de estudio se difunden.

Uno de los primeros problemas que nos encontramos para llegar a cumplir esta pretensión histórica tiene que ver con los escalones educativos previos a los "altos estudios". Más de 960.000 argentinos nunca fueron a la escuela, según surge del último censo del Instituto de Estadística y Censos (Indec) ${ }^{1}$. Dado que en el país existe un total de 1.142.151 graduados universitarios, se advierte que hay casi un analfabeto puro por cada egresado.

A este cuadro se suma otro fenómeno preocupante: 3.695 .830 argentinos nunca terminaron la educación primaria y pasan a integrar, por lo tanto, la categoría de analfabetos funcionales, es decir, en general tienen serias dificultades para comprender lo que leen, al tiempo que no pueden expresarse con claridad tanto en forma verbal como por escrito.

Aunque el número de los que nunca tuvieron contacto con el sistema educativo tiende a descender, los analfabeto funcionales es un grupo que registra un ligero crecimiento en los últimos 10 años.

Algunos autores -Cecilia Braslavky, Juan Carlos Tedesco- hablan también de "analfabetismo académico", es decir, el de aquellos que al intentar ingresar en la Universidad no pueden comprender lo que leen, expresarse, argumentar o entender una consigna. Desde 1989 no existen en el país políticas nacionales para enfrentar el fenómeno del analfabetismo, que quedó así sujeto a iniciativas aisladas, impulsadas por algunos gobiernos provinciales y organizaciones sociales y comunitarias.

Sin planificación, conviven experiencias disímiles de alfabetización, que ya no se agotan en enseñar a leer, escribir y calcular. Consiste en preparar a una persona para que regrese al sistema educativo, devolverle confianza en sus saberes, conciencia de sus derechos, manejo de la tecnología y una serie de habilidades para tomar decisiones de manera autónoma.

\section{EL INGRESO EN LAS UNIVERSIDADES ARGENTINAS}

\subsection{Matrícula en el Período 1950-2000}

Según los datos publicados por al Secretaría de Políticas Universitarias del Ministerio de Educación, Ciencia y Tecnología, la matrícula global de la educación superior -cantidad de alumnos totales del sistema

\footnotetext{
${ }^{1}$ Citado por el Diario Nación en su edición del día 8 de agosto de 2004.
} 
en un año determinado-, ha crecido fuertemente en el período. Se multiplicó por 20, pasando de 86.080 estudiantes en 1950 a 1.724 .397 en el año $2000^{2}$

En el año 1950 la matrícula era casi solamente de las universidades nacionales ya que la no universitaria era prácticamente mínima -5635 estudiantes- y las universidades privadas se autorizarían luego en el 58.

En 1970 aparece una participación mayor de la universidad privada (12,5\%) y de la no universitaria(13,5\%) frente al $74 \%$ de la universidad nacional. Vale destacar que el bajo crecimiento de la matrícula nacional está fuertemente determinado por las políticas de la dictadura militar instaurada en 1966, con su secuela de vaciamiento de las universidades nacionales y restricciones del ingreso.

En 1975 la matrícula de las universidades nacionales era de 431.454 estudiantes mayor que la de 388.101 estudiantes de 1980, marcando claramente el efecto represivo de la dictadura militar (1976-1983). En ese mismo sentido la participación de las universidades nacionales bajó del $78 \%$ en 1975 al $66 \%$ en 1980 y se incrementó la de las privadas del $11 \%$ al $15 \%$ y la de la educación superior no universitaria del $11 \%$ al $19 \%$.

Entre 1985 y 2000, el crecimiento de la matrícula fue casi explosivo, superando a cualquier otro período de la historia argentina, pasando de 777.488 alumnos en 1980 a 1.724 .397 en el año $2000 .^{3}$

\section{Crecimiento de la matrícula total de la educación superior (1950-2000)}

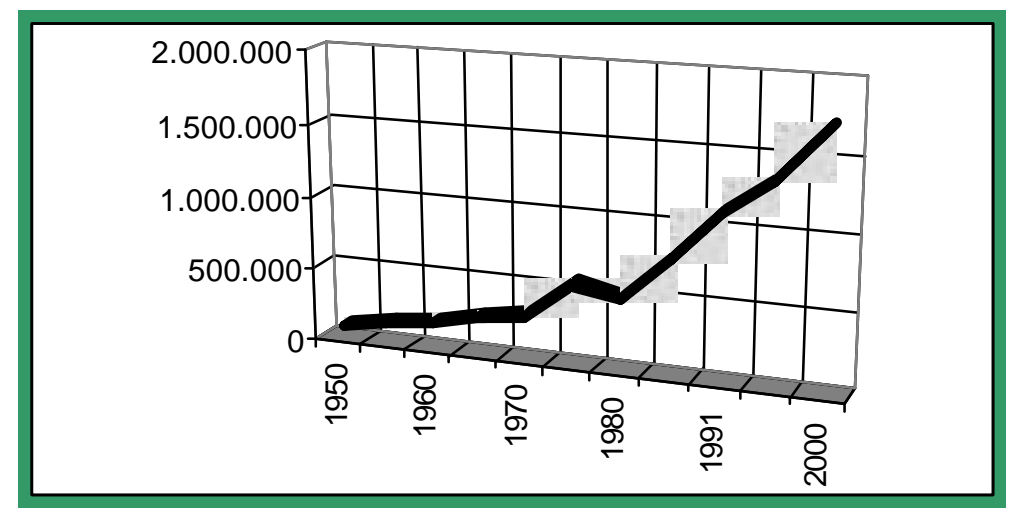

Podemos señalar como alguna de las causas de ese aumento en el crecimiento global de la matrícula:

- El crecimiento de la oferta tanto en universidades públicas, privadas y de educación superior no universitaria. Es destacable el impacto territorial de este crecimiento, llevando ofertas de educación superior a ciudades pequeñas del interior del país o multiplicando ofertas existentes. Además de la creación de nuevas carreras, multiplicación y superposición de ofertas en los grandes conglomerados urbanos como el Gran Buenos Aires, lo que hacía más atractiva la posibilidad de continuar estudios superiores.

- La apertura social y cultural que significó la democracia en relación con el miedo y el control ideológico generado en la dictadura. En este sentido es notorio la reapertura (en algunos ca-

\footnotetext{
${ }^{2}$ Datos oficiales citados por Fernández Lamarra, Norberto en La Educación Superior Argentina en Debate, pag 103.

${ }^{3}$ Ibidem. Pag 104.
} 
sos) y el crecimiento de las carrera de artes y ciencias humanas y sociales muchas cerradas en la dictadura militar.

- La creciente exigencia del mundo laboral paralelo al crecimiento del desempleo, que impulsa a muchos jóvenes a intentar la obtención de un título para acceder a alguno de los ultra restringidos lugares dentro del mundo del trabajo. ${ }^{4}$

\section{$2.2 \quad$ El crecimiento desde 1983 en adelante}

Analizando el cuadro anterior notamos el crecimiento desde la recuperación democrática. En el período 1985-2000 es notable el crecimiento de la matrícula de la educación superior en su conjunto: 122\%. El mayor crecimiento en el período lo registró la superior no-universitaria $(150 \%)$, las universidades nacionales crecieron un $114 \%$ y las privadas un $107 \%$.

Ahora bien, estas cifras aparentemente espectaculares, tienen también sus variaciones, en especial la de las universidades nacionales. Por ejemplo esta definición está seriamente afectada por la definición de lo que se considera alumno. Si se aplicase en forma rigurosa lo que establece la Ley de Educación Superior (más allá del juicio que esto nos merezca) en su artículo 50, las universidades nacionales deberían considerar sólo alumno al que curse con regularidad sus estudios y por lo tanto apruebe, al menos 2 materias por año.

Cada universidad tiene interpretaciones diferentes sobre lo que se considera alumnos. Marta Kisilevsky, responsable del Programa de Mejoramiento del Sistema de Información Universitaria desde su inicio hasta el 2001, señala que se ha tratado durante mucho tiempo de llegar a un acuerdo respecto a la definición de alumno y que todavía está en discusión, si bien se han registrado avances ${ }^{5}$

Augusto Pérez Lindo ${ }^{6}$ plantea como interrogante si la expansión universitaria es real o ficticia, ya que ha crecido la matrícula fuertemente pero el número de graduados se ha mantenido casi constante en los últimos años (cómo analizaremos más adelante).

Pérez Lindo afirma que hay dos opciones: corregir la visión de lo que parece crecimiento y no lo es, a partir de un análisis crítico, o seguir sosteniendo que sí se crece.

\section{ANÁLISIS SOCIAL, ECONÓMICO Y CULTURAL DE LOS ESTUDIANTES}

Mario Toer $^{7}$ realizó varias investigaciones acerca de los estudiantes del la Universidad de Buenos Aires. El perfil de los estudiantes de la UBA muestra que el 12,5\% son de nivel socieconocomico alto; $35,5 \%$ medio $A ; 39,5 \%$ medio $B$; y $12,7 \%$ bajo. Es decir el $75 \%$ son de clase media -con leve predominio de la clase media baja- y el $12 \%$ son de clase alta o baja. Estas cifras, y otros estudios similares en otras universidades argentinas, dan cuenta de la preponderancia de aquellos sectores sociales denominados "medios" en la composición del claustro estudiantil de las universidades públicas.

\footnotetext{
${ }^{4}$ En este sentido ver el trabajo de Marcela Mollis sobre "Universidades Argentinas. Identidades Alteradas", publicado por EUDEBA en el año 2202.

${ }^{5}$ Kisilevsky, Marta, Condiciones Sociales y Pedagógicas de Ingreso a la Educación Superior.

${ }^{6}$ Citado por Fernández Lamarra,, Norberto, La Educación Superior Argentina en Debate, pag 107.

${ }^{7}$ Ver Toer Mario, "Cómo son los estudiantes. Perfil socioeconomico y cultural de los estudiantes de la UBA", ECA -Catológos, Buenos Aires, 1998 y Toer, Mario, "El perfil de los estudiantes y el rendimiento académico", Bs. As,2001.
} 
Otro aspecto importante, que se desprende del mismo estudio, es que el $60 \%$ trabaja mayoritariamente en jornada completa. El resto no trabaja (40\%) o busca trabajo (16\%).

\section{Distribución por estrato de estudiantes de la UBA. (Toer)}

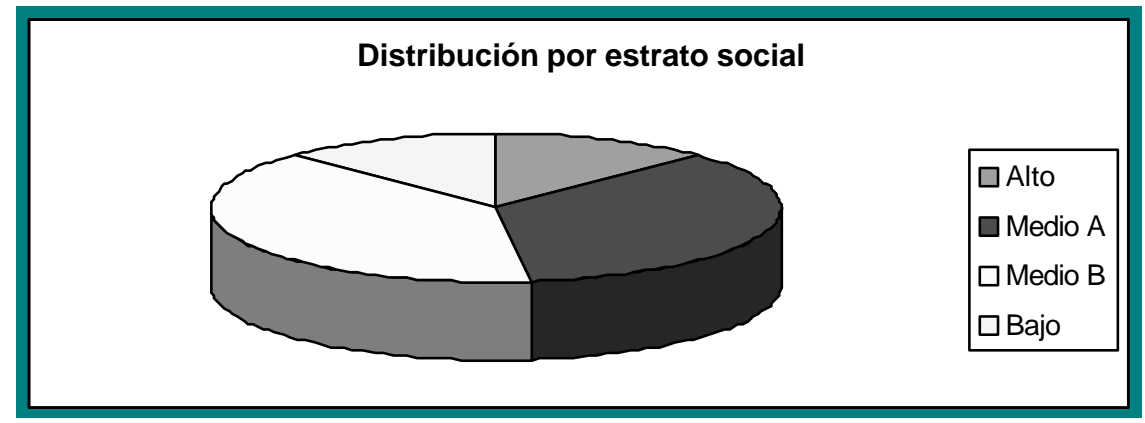

Fuente. Toer Mario, "Cómo son los estudiantes. Perfil socioeconómico y cultural de los estudiantes de la UBA", ECA-Catológos, Buenos Aires, 1998 y Toer, Mario, "El perfil de los estudiantes y el rendimiento académico", Bs. As,2001.

Según diversos estudios la relación estudio-trabajo en la Universidad de Buenos Aires puede ser considerada representativa de los estudiantes universitarios argentinos: la mayoría trabaja en jornada completa en tareas que tienen poco o nada que ver con lo que estudian. Según el autor esto contribuye como una de las causas de abandono y 'lentificación' de los estudios superiores.

Otro dato es el sostenido incremento en los últimos 20 años del ingreso de alumnos al subsistema universitario con relación a la población en edad. Entre 1980 y 2000 la participación de los estudiantes de 18 años en el sector universitario pasó de representar el 12,4\% al 52\%, ascendiendo a 350.000 el número de nuevos inscriptos en el año 2000. Asimismo, es interesante observar las expectativas de los estudiantes del nivel medio respecto de la continuación de sus estudios. Ocho de cada 10 estudiantes de nivel medio piensan continuar estudios de nivel superior: un $60 \%$ tiene intención de seguir carreras universitarias y un $20 \%$ carreras cortas $^{8}$.

\subsection{Egreso y Deserción}

A la creciente intención de continuar estudios universitarios se le contrapone uno de los principales problemas que enfrentan hoy las universidades: la deserción. Los datos muestran que entre 1997-2000 hubo importantes diferencias entre el número de nuevos alumnos inscriptos en el primer año de las carreras y los reinscriptos al segundo año en el año siguiente. Comparando los datos resulta que entre 1997 y 1998 el porcentaje de reinscriptos representó el 60\%; entre 1998 y 1999 el 63\% y entre 1999 y 2000 , el 61\%. En consecuencia, podríamos decir que se desconoce el destino de casi un $40 \%$ de los alumnos inscriptos en los primeros años de las carreras al año de haber ingresado a la universidad. ${ }^{10}$

Estas tendencias pueden ser explicadas, en parte, por el alto grado de complejidad alcanzado por el sistema, así como por el proceso de especialización producido en sus dimensiones de gobierno y gestión, los cuales han generado una dinámica de diferenciación y segmentación. Como consecuencia, los estudian-

\footnotetext{
${ }^{8}$ Kisilevsky, Marta, "Condiciones Sociales y Pedagógicas de Ingreso a la Educación Superior" , Pag. 158.

${ }^{9}$ Información provista por el Programa de Mejoramiento del Sistema de Información Universitaria (PMSIU) de la Secretaría de Políticas Universitarias. Citada en la página web www.mcye.edu.ar

${ }^{10}$ Información provista por la Secretaría de Políticas Universitarias del MCyE, citado en www.mcye.edu.ar
} 
tes se enfrentan con un sistema desarticulado y con caminos terminales al momento de decidir por estudios de nivel superior. De esta forma se dificulta tanto la movilidad en el nivel institucional -es decir entre distintas carreras de una misma institución- cuanto en el nivel interinstitucional -ya sea entre iguales o diferentes carreras de distintas instituciones.

Los datos muestran las dificultades ocasionadas a los estudiantes en su conjunto: a los del sector no universitario cuyos estudios son terminales porque no cuentan con sistemas de acreditación que les permitan una circulación fluida hacia las universidades; y también se ven afectados los alumnos universitarios que inician sus estudios en las universidades y que deciden continuarlos en un terciario, o en otra carrera dentro del mismo nivel universitario.

La evolución del número de egresados universitarios en los últimos años muestra, para universidades nacionales, que el total se mantiene relativamente constante en alrededor de 34.000/35.000 egresados anuales. Esto pone en evidencia una evidente disparidad con el crecimiento del número de ingresantes antes mencionado.

En cuanto a las universidades privadas el número de egresados es creciente a partir de 1995, en el que han comenzado a graduarse los estudiantes de las nuevas creadas a inicios de los 90 . Es de señalar que mientras en lo referido a estudiantes matriculados, las universidades privadas tienen alrededor del $10 \%$ del total, en egresados esta proporción es mucho mayor: ha estado oscilando entre el $20 \%$ y el $25 \%$. Esta información muestra que en las universidades privadas la relación número de estudiantes/número de egresados es muy superior a la que existe en las universidades nacionales.

Cantidad de egresados en las Universidades Argentinas para el período 1991-97.

\begin{tabular}{|c|c|c|}
\hline Años & Univ. Nacionales & Univ. Privadas \\
\hline 1991 & 32323 & 8212 \\
\hline 1992 & 32296 & 8044 \\
\hline 1993 & 36964 & 8571 \\
\hline 1994 & 35995 & 8241 \\
\hline 1995 & 35546 & 9376 \\
\hline 1996 & 34675 & 9635 \\
\hline 1997 & 34503 & 11260 \\
\hline
\end{tabular}

Egresados en las Univer. Públicas 80-90

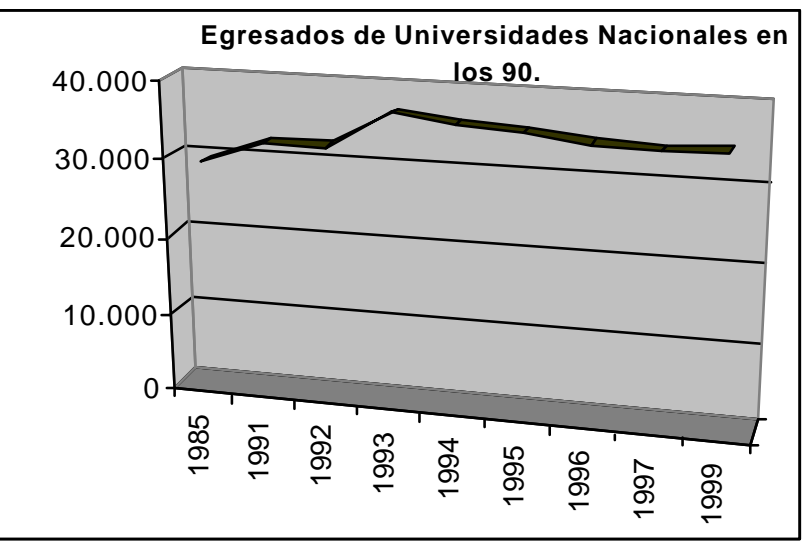

Matrícula en las Univer. públicas 80-90

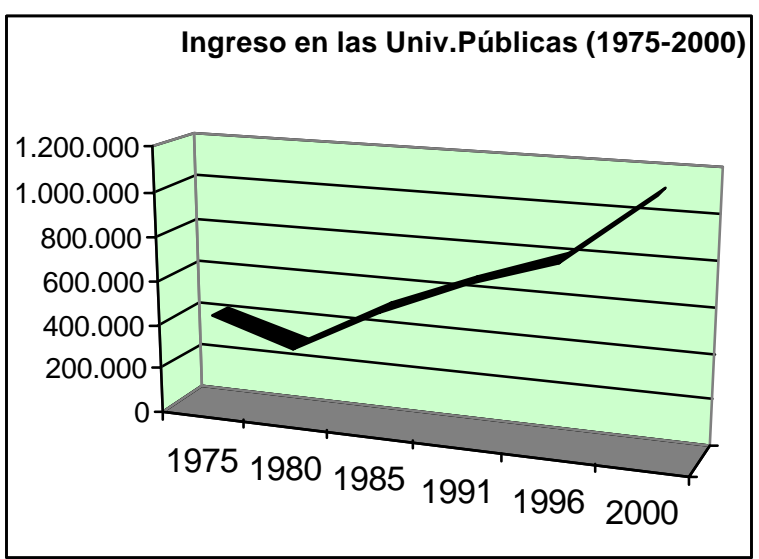


Los gráficos anteriores muestran la evolución de la curva de matrícula total de las universidades nacionales, que como se ha dicho registra un importante crecimiento desde 1983 en adelante. Al mismo tiempo la tasa de graduación permanece casi constante entre 1985-1999, es decir cuando debieron haberse graduado muchos de los ingresantes anteriores. Estos graduados son del sistema universitario en su conjunto, con mayoría de carreras de 5 años, pero incluyendo también algunas ofertas de 2 o 3 años, como es el caso de las Tecnicaturas que ofrecen varias universidades nacionales.

Es significativa la estabilidad de la tasa de graduación, a pesar del crecimiento casi constante del número de ingresantes.

\section{ALGUNAS CONSIDERACIONES SOBRE LA PERMANENCIA EN LA UNIVER- SIDAD}

Si tomáramos los datos mencionados como indicadores de situación, debiéramos estar pensando en la necesidad imperiosa de continuar interrogándonos y reflexionando para analizar la realidad y generar políticas globales, estrategias superadoras y procesos transformadores para mejorar el rendimiento académico de los alumnos en la Universidad haciendo converger este eje con la discusión política relativa a los mecanismos de ingreso y las políticas institucionales instrumentadas para fortalecer el mejoramiento de la enseñanza de grado.

Si pensamos el ingreso en relación con la permanencia, podemos decir que las opiniones se dividen entre sectores que sostienen que el ingreso irrestricto garantiza per se las condiciones de igualdad y de equidad y entre quienes sostienen que "no obstante las excepciones y los cambios más recientes, los resultados del ingreso irrestricto que ha operado de hecho en la mayoría de las universidades públicas de Argentina en los años setenta y ochenta, ha generado una situación extremadamente desventajosa con relación al alto índice de deserción y de duración media de las carreras"11. Sin embargo, un análisis más profundo de la situación revela que -simultáneamente a los "índices de bajo nivel de aprendizaje" en los estudiantesdebe reconocerse también la existencia de condiciones sociales y económicas -empleos inestables, descenso de los ingresos del grupo familiar, etc- como fuertes condicionantes a la hora de continuar una carrera universitaria.

El segundo dato importante es la diferencia entre el capital simbólico exigido para permanecer como alumno regular en la universidad y el capital que los estudiantes han podido construir en su paso por anteriores niveles del sistema educativo. Diversos autores -José Luis Coraggio, Martha Kieslevky, Marcela Mollis $^{12}$ - ubican a esta características del sistema educativo argentino como una de las causas de la deserción en los dos primeros años de la mayoría de las carreras universitarias.

En este sentido, debe tomarse en consideración la situación reconocida en Argentina con relación a las dificultades de una masa grande de estudiantes provenientes del nivel secundario, para superar pruebas de aptitudes para el ingreso a la universidad, tal como lo prueban los resultados de las evaluaciones diagnósticas administradas a los ingresantes a la Universidad. Piffano sostiene en relación con esta situación que "La reacción generalizada que se advierte sobre este fenómeno, es la de formular un juicio negativo

\footnotetext{
${ }^{11}$ Piffano, H, "La Universidad y su financiamiento". En La Universidad ahora. № 11-12. UBA. Primer semestre '99. Pág. 116.

${ }^{12}$ Ver, entre otros, el trabajo de Marcela Mollis “ Un breve diagnostico de las universidades argentinas: Identidades Alteradas”, publicado en versión digital en la página web de la Secretaría de Políticas Universitarias del Ministerio de Cultura, Ciencia y Tecnología de la Nación
} 
respecto de los logros obtenidos en la enseñanza de los niveles primario y secundario, sin advertir suficientemente, sin embargo, la existencia de situaciones diferenciales que es posible encontrar entre jóvenes pertenecientes a familias con diverso nivel de desarrollo humano relativo. Lo que intentamos señalar aquí es que los condicionantes socioeconómicos implican la creación de barreras académicas o intelectuales muy difíciles de superar al momento de alcanzar la edad escolar universitaria... castigando en mayor medida a las franjas con menor desarrollo humano, las que naturalmente coinciden con los grupos de familias más pobres". 13

Al mismo tiempo se debería reconocer la necesidad de mejorar aquellos factores que hoy limitan la posibilidades académicas de los estudiantes universitarios, asumiendo como centrales aquellos que hacen a la deserción, la repetición, la duración de las carreras y los mecanismos de evaluación, en tanto estos factores internos afectan al impacto externo de la institución universitaria en función de su posibilidad de respuesta a las demandas y necesidades estratégicas del conjunto de la sociedad en la que está inserta y el impacto que se pretende lograr en el medio socio-económico y cultural, a partir de la generación e inserción de sus graduados y el desarrollo de sus programas y proyectos científicos, tecnológicos, educativos, sociales, económicos y culturales. Asimismo, debemos reconocer también que la equidad social se vincula no sólo con igualdad de oportunidades en el interior del sistema universitario, sino con factores exógenos como son el conjunto de políticas socioeconómicas que impactan en la distribución del ingreso y en la Universidad misma como una parte indisoluble del sistema social ${ }^{14}$.

Como ya se ha mencionado diversos autores afirman que "la exclusión de un elevado porcentaje de la población estudiantil proviene de etapas educativas previas a la universitaria y en la gran mayoría de los casos la razón es económica" ${ }^{15}$, razón por la cual debe tenerse en cuenta que la desigualdad de la calidad educativa en los niveles previos a la educación superior es uno de los factores productores de deserción, sobre todo en estratos de población de menores recursos. A ello debe agregarse el fenómeno, potenciado en los años noventa de una política educativa global condicionada por una baja asignación presupuestaria para el sostenimiento de la educación pública, que no ha hecho sino generar y aumentar la fragmentación del subsistema de educación de nivel medio y la existencia de circuitos de formación pedagógica diferenciados sobre la base de las desigualdades socioeconómicas y culturales de su propia población estudiantil.

Este hecho puede ponerse de manifiesto a partir de algunos ejemplos evidentes, que pueden $\mathrm{d}-$ servarse en una franja que en sus extremos va desde el fomento de las políticas sociales compensatorias en detrimento de proyectos pedagógicos justamente en instituciones en que su población más necesita de estas últimos para generar sus conocimientos y movilizar estímulos previos-, hasta la existencia de sistemas de doble escolaridad en escuelas privadas que, naturalmente, potencian las capacidades, habilidades y conocimientos de alumnos que ya de por sí poseen un plus diferencial frente a los primeros, lo cual confirma, desde esa fragmentación del sistema, la heterogeneidad y variabilidad de la población que potencialmente ingresa a las universidades, en condiciones de desigualdad estructural previa.

\footnotetext{
${ }^{13}$ Piffano, Ibidem Pág. 114.

${ }^{14}$ Ver: Hidalgo, J.C., "El financiamiento universitario". En: Pugliese, J.C.(editor): Políticas de Estado para la Universidad Argentina" .Ministerio de Educación, Ciencia y Tecnología.

${ }^{15}$ Ibidem, pag. 47.
} 


\section{EXPECTATIVAS DE LOS JÓVENES RESPECTO A LOS ESTUDIOS SUPERIO- RES}

En nuestro país existe una importante cantidad de jóvenes que tienen aspiraciones de realizar estudios de nivel superior Sin embargo, esas aspiraciones en muchos casos no logran concretarse, seguramente por múltiples factores de índole subjetiva, pero también por muchos factores de índole social y económico, desde donde será deseable oportunamente pensar estrategias de intervención.

Algunos estudios como el realizado por Marta Kiselvsky y Cecilia Veleda ${ }^{16}$, sobre las aspiraciones de los estudiantes de los últimos años del secundario muestran esta tendencia a seguir estudios superiores. Como parte de su investigación Kiselvky y Veleda elaboraron un cuestionario que fue respondido por más de 300.000 jóvenes de los dos últimos años del nivel medio de todo el país, de los cuales solamente el $7 \%$ expresó no saber qué hacer el año siguiente y un $8,4 \%$ no respondió la pregunta sobre sus deseos futuros.

- Las opciones consultadas fueron:

- Estudiar un curso/carrera corta

- Estudiar una carrera universitaria

- Trabajar y estudiar un curso/carrera corta

- Trabajar y estudiar una carrera universitaria

- Sólo trabajar

- No sabe

Según el resultado del trabajo, es posible observar que ocho de cada 10 estudiantes de nivel medio piensan continuar estudios de nivel superior: un $60 \%$ en el nivel universitario y un $21 \%$ encarando un curso o carrera corta. También es interesante notar que aquellos que se autoexcluyen de la posibilidad de continuar estudios y optan por trabajar son sólo el 3\% del total.

Expectativas de los jóvenes de los últimos años de la educación media

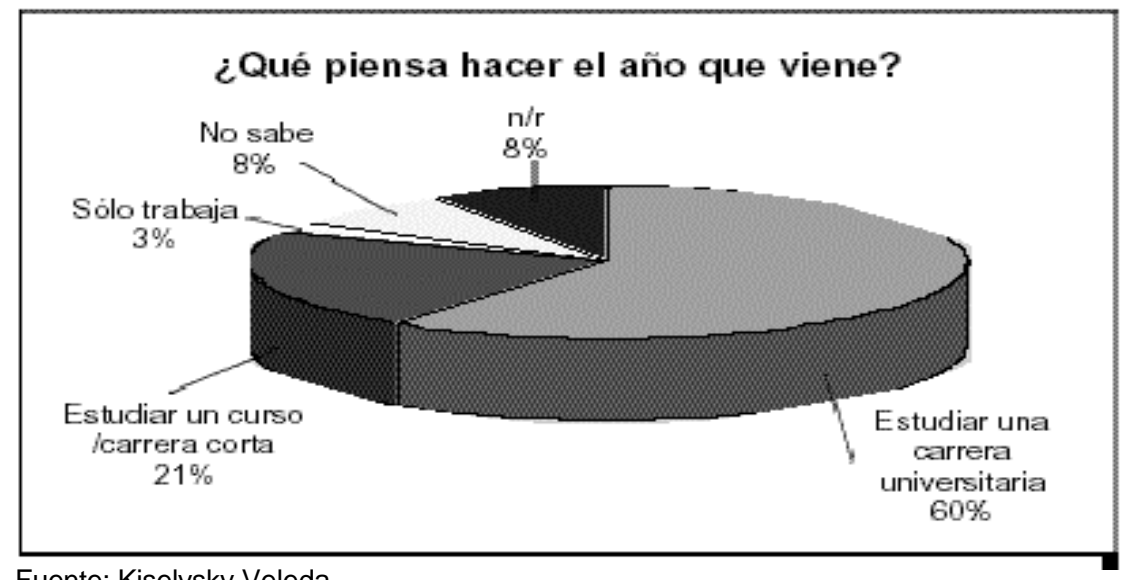

Fuente: Kiselvsky-Veleda.

${ }^{16}$ Kisilevsky, Marta y Veleda, Cecilia , "Condiciones Sociales y Pedagógicas de Ingreso a la Educación Superior" , Publicación digital en la página web de la SPU del Ministerio de Educación Ciencia y Tecnología 
Que este estudio y otros realizados sobre el tema den parecidos resultados ${ }^{17}$, muestra que desde 1983 hasta nuestros días la gran mayoría de los jóvenes que finalizan la educación media quiere seguir estudios superiores.

Siguiendo con la investigación mencionada, otro dato importante es el perfil de los estudiantes. Con datos provenientes de la Encuesta Permanente de Hogares (EPH) se mostró la existencia de perfiles de estudiantes diferenciados muy claramente entre sí. En primer lugar, las investigadoras analizaron los rasgos típicos de los alumnos de institutos terciarios y de universidades e indagaron en algunos aspectos vinculados con su origen social y educativo. La escuela secundaria de procedencia así como el traslado de ciudad para estudiar son dos indicadores que ponen de manifiesto la existencia de circuitos por los que transitan los estudiantes. Un dato importante que complementa las razones individuales de la elección de estudios superiores es que la oferta académica en su dimensión geográfica es susceptible de ejercer un impacto en las orientaciones

Así, la distribución espacial de las distintas carreras de enseñanza superior tiene como consecuencia que todos los estudiantes (según su lugar de residencia) no se enfrenten con la misma capacidad de afrontar los costos relativos para el acceso a las diferentes opciones. Algunos estudios empíricos muestran que los costos directos de la educación que recaen sobre los estudiantes son notablemente más elevados si el estudiante realiza estudios superiores fuera de su lugar de origen.

En este sentido, los datos socioeconómicos tienen un peso considerable en la definición de los perfiles, como puede verse a través de los ingresos de los hogares a los que pertenecen.

Se vio como los estudiantes de nivel no universitario circularon por la universidad antes de ingresar a los institutos en un porcentaje considerable, por lo que es posible pensar en un fenómeno acumulado a lo largo de muchos años, a partir del cual se generó una construcción social de los estudios terciarios como una "segunda opción", categoría que en no pocos casos contiene -aunque implícito-, un juicio de valor negativo. Las propias visiones de los futuros estudiantes como "estudios de primera y de segunda" remiten a este fenómeno

Los que quedan en su lugar de residencia deben elegir quizás lo que menos les desagrada, a partir de lo cual pueden explicarse los problemas de deserción. En otros casos, en los que hay ofertas más variadas por parte de las universidades, la explicación puede ser otra. Por ejemplo, que las expectativas de inserción laboral más rápida, y la imagen (y realidad) de la extensa duración de una carrera universitaria, tengan un peso significativo en el momento de decidir no continuar con los estudios. Los sectores sociales más altos prefieren la universidad por su nivel académico, la exigencia, la formación amplia, el reconocimiento en el mercado laboral y el prestigio social, mientras que los jóvenes de sectores más bajos prefieren las carreras de corta duración, por ser menos costosas, posibilitar rápidas salidas al mercado laboral y dar la posibilidad de trabajar y estudiar al mismo tiempo.

También fue posible, a partir de la revisión de las investigaciones producidas sobre el tema, comprobar la insatisfacción que presentan las instituciones de nivel superior respecto del nivel previo de formación de sus ingresantes. En muchos casos se lo planteó como "el" problema: la baja calidad académica de los estudiantes de nivel medio. En este sentido, si bien acordamos en que la ampliación y diversificación de

\footnotetext{
${ }^{17}$ Por ejemplo una encuesta realizada por el diario Clarín sobre 9.000 alumnos del último año de la educación media.
} 
la oferta de estudios de nivel superior parece seguir siendo algo necesario, también lo es la orientación previa a la elección de los estudios.

Las intervenciones en ese terreno son necesarias y deben estar sujetas permanentemente a revisión, puesto que son constantes los desajustes entre oferta y demanda. Sin embargo, no es posible dejar de considerar los esfuerzos que muchas instituciones están haciendo para responder a la demanda con criterios racionales al interior de cada una. Aun así, nos seguimos encontrando con una realidad que es contundente: los que demandan educación superior son cada vez más, y los mecanismos de apoyo y el desajuste entre el nivel medio y el superior aún son débiles.

\section{ALGUNAS REFLEXIONES PROVISORIAS}

Se debe señalar, en el caso de Argentina, la notoria ausencia de políticas específicas dirigidas a aumentar los índices de retención de la población en el nivel. El problema no parece radicar tanto en las dificultades en el acceso, sino más bien en factores institucionales que impiden a los estudiantes permanecer en el sistema. Sigue siendo un tema controvertido el de colocar pruebas de ingreso y el impacto que sus resultados tienen.

Desde visiones marcadamente economicistas se deduce que el problema es que hay demasiados alumnos y se proponen exámenes de ingresos, cupos, arancelamiento con sistemas de becas, etc. Como se ha dicho, la aplicación de esta última propuesta sugiere una notable contradicción en el hecho de que un sistema de becas (basado en el mérito) ofrecido recién en el nivel universitario deja afuera una parte de la población que posiblemente haya transitado por un sistema altamente segmentado de escolaridad básica y que ingresaría con un capital cultural deficitario para tener éxito en la educación superior. De ahí la importancia de ver el problema desde su origen, es decir, desde el momento en que la población escolar se encuentra atravesando sus años de escuela básica.

Mucho habría que mejorar respecto de la tan difundida idea de la articulación entre la escuela media y el nivel superior. $L$ a preparación académica debería comenzar hacia los primeros años del nivel medio De todas formas, es necesario realizar estudios de seguimiento de estudiantes (al menos con cohortes teóricas) para conocer los factores predictivos del éxito o fracaso y poder tomar decisiones al respecto con información más certera acerca de los aspectos culturales (familiares, de contexto) que inciden en el camino hacia los estudios de nivel superior. También habría que dirigir la atención al valor de los métodos estadísticos avanzados que identifican los efectos netos de cada uno de estos factores.

Frente a ello, y ante estos condicionamientos, no debe centrarse la Universidad en la discusión sobre la promoción de un sistema de ingreso restricto para superar una diversidad, una heterogeneidad y una desigualdad externa previa que impacta en el ingreso al sistema universitario, sino considerar la necesidad de desarrollar políticas educativas para facilitar la orientación, la continuidad, el seguimiento y el apoyo para una retención con calidad, por un lado, y para contribuir a una más adecuada transición entre el nivel medio y el universitario, por otro. Ello porque sostenemos la convicción de que la educación pública juega un papel central en cualquier sociedad que valore la democracia y la justicia social, razón por la cual apoyarla se convierte en una estrategia política de desarrollo clave para asegurar que, pese a la situación económica y sociocultural de origen, cualquier estudiante pueda educarse para superar las condiciones de desigualdad originarias. 
En el caso de Argentina, de acuerdo a los datos cuali y cuantitativos trabajados podemos establecer algunas conclusiones, siempre provisorias:

- Existe una población de educación superior que crece a un ritmo infinitamente mayor que el crecimiento que experimentan los egresados del nivel, especialmente universitario.

- No se implementan política nacionales, y son muy incipientes las institucionales que tiendan a facilitar el acceso y la permanencia de los estudiantes en el nivel superior.

- Las consecuencias de prolongar una situación de tan corta estadía de los estudiantes en el nivel superior de educación no han sido objeto de investigación, es decir, no se conoce el impacto del tránsito de los estudiantes por las aulas de las instituciones de educación superior, ni desde el punto de vista del sujeto ni de las propias políticas educativas.

- Tampoco hay estudios que midan qué efecto (social, educativo y económico) produciría la puesta en práctica de políticas de mejoramiento de la retención en los primeros años del nivel superior.

- Para muchos actores de la comunidad universitaria el problema de la deserción está siendo "naturalizado", de forma tal que resulta "lógico" que los estudiantes empiecen a desaparecer de las aulas hacia comienzos del cuatrimestre y se estabilice su número en los meses subsiguientes.

En resumen, la responsabilidad sobre la educación superior necesita que se la someta a revisión al menos en tres frentes:

- En primer lugar, es notorio el impacto que las condiciones materiales de vida tienen sobre las posibilidades de ingreso y permanencia en la educación superior. Tal es el caso de la pobreza, la caída del ingreso familiar o, el desempleo que actúan como fuertes determinaciones en todos los niveles educativos e impiden a millones de jóvenes acceder a una titulación universitaria.

- En segundo lugar se debería reconocer la disparidad de capital simbólico que los diferentes sujetos sociales portan al momento de ingresar a los estudios superiores. Como ya se ha analizado este capital está fuertemente determinado por su pertenencia a una clase social determinada. Esta diferencia de capital cultural, en el sentido de poseer un activo cultural diferente al exigido por la formalidad académica, condiciona fuertemente el ingreso y la permanencia en loa estudios superiores.

- En último lugar es muy difícil para las instituciones de educación superior suplir las fuertes ausencias que los niveles educativos anteriores dejan en la formación de los jóvenes, las cuales se ha profundizado con la transformación educativa de mediados de los noventa. La inclusión en los estudios superiores (o la posibilidad de ella) debería comenzar en la escuela básica.

\section{CAMINOS}

A riesgo de esquematizar podríamos anticipar dos caminos para las universidades en la República Argentina. 
El primero de ellos es una universidad pequeña, cerrada a una clase social, gobernada por la elite de intelectuales que posea el capital simbólico más adecuado para defender y sostener el poder de la clase dominante, carente de conflictos y eficiente en los parámetros de la ideología neoliberal. La que seguramente será más organizada y silenciosa que la actual.

La otra posibilidad sería una universidad policlasista, que reconozca y se haga cargo de desigualdades previas, democrática en su gobierno, abierto a los conflictos sociales que la atraviesan y con índices de inclusión social creciente que distribuya el conocimiento ampliamente, con la decidida pretensión de usarlo como factor que ayude a la igualdad social.

La sociedad que deseamos incluirá en su gestación a los claustros universitarios, al tiempo que los transformará para que nazca una nueva universidad. La sociedad de nuestras peores pesadillas, también.

\section{BIBLIOGRAFÍA}

Luis y Adolfo Vispo (coord.). Bs. As. Consejo Interuniversitario

Nacional/Miño y Dávila Editores. UNGS. 2001

CONSEJO INTERUNIVERSITARIO NACIONAL, Contribución al estudio del sistema universitario argentino, .Buenos Aires, Miño y Dávila Editores, 2001

CONSEJO INTERUNIVERSitARIO NACIONAL, Argentina 2020. Manifiesto por la Educación Pública, Setiembre 2001, Bs. As.

CORAGgIo, JOSÉ L, Política social y economía del trabajo Buenos Aires,

UNGS-Miño y Dávila. 1999.

CORAGGIO, JOSÉ LUIS Y ADOLFO VISPO (coord.), Contribución al estudio

del sistema universitario argentino, Buenos Aires, Consejo Interuniversitario

Nacional/Miño y Dávila Editores. 2001

FERNÁNDEZ LAMARRA, Norberto. La educación superior argentina en debate, Eudeba- IESALC, 2003.

FIEL-CEP , Una educación para el siglo XXI: el caso argentino y otras experiencias internacionales. Buenos Aires. 2001.

IAZZETTA, Osvaldo M. La recreación de la dimensión pública de la

Universidad, en Pensamiento Universitario .Buenos Aires. Año 9, № 9, A b r i l . 2001.

KISILEVSKY, Marta y VelEDA, Cecilia, Condiciones Sociales y Pedagógicas de Ingreso a la Educación Superior, Publicación digital en la página web de la SPU del Ministerio de Educación Ciencia y Tecnología, Buenos Aires, 2002.

PRIETO CASTILlo, Daniel, La institución universitaria, Mendoza, EDIUNC, 1997.

PUGLIESE (h), Juan Carlos, Cambiar la universidad para mejorar la

Sociedad, en Pensamiento Universitario (Buenos Aires) Año 9, № 9, Abril. 2001.

TOER, Mario, Como son los estudiantes. Perfil socioeconomico y cultural de los estudiantes de la UBA, ECACatológos, Buenos Aires, 1998. 


\title{
Contactar
}

Revista lberoamericana de Educación

\author{
Principal OEI
}

